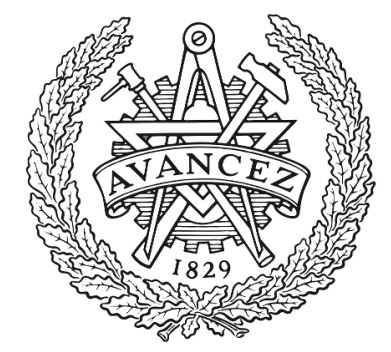

\title{
CHALMERS
}

UNIVERSITY OF TECHNOLOGY

\section{State of Power Prediction for Battery Systems with Parallel-Connected Units}

Downloaded from: https://research.chalmers.se, 2023-04-26 12:23 UTC

Citation for the original published paper (version of record):

Han, W., Altaf, F., Zou, C. et al (2022). State of Power Prediction for Battery Systems with

Parallel-Connected Units. IEEE Transactions on Transportation Electrification, 8(1): 925-935.

http://dx.doi.org/10.1109/TTE.2021.3101242

N.B. When citing this work, cite the original published paper.

C2022 IEEE. Personal use of this material is permitted.

However, permission to reprint/republish this material for advertising or promotional purposes 


\title{
State of Power Prediction for Battery Systems with Parallel-Connected Units
}

\author{
Weiji Han, Member, IEEE, Faisal Altaf, Member, IEEE, Changfu Zou, Member, IEEE \\ and Torsten Wik, Member, IEEE
}

\begin{abstract}
To meet the ever-increasing demand for energy storage and power supply, battery systems are being vastly applied to, e.g., grid-level energy storage and automotive traction electrification. In pursuit of safe, efficient, and cost-effective operation, it is critical to predict the maximum acceptable battery power on the fly, commonly referred to as the battery system's state of power (SoP). As compared to the SoP prediction at the battery cell level, predicting the SoP of a multi-battery system, especially including parallel-connected cells/modules/packs, is much more complicated and far less investigated. To solve this problem, a system-model-based SoP prediction method is first proposed in this paper. Specifically, based on the formulated system model and generic state-space representation, the challenge of nonmonotonic system state evolution, arising from the dynamic parallel current distribution, is identified and systematically addressed by the proposed method. As demonstrated by tests on a battery system set up with experimentally verified parameter values, the proposed method outperforms the commonly applied cell-SoP based methods for providing a more accurate and reliable prediction of the battery system SoP. Moreover, the proposed prediction framework presented in generic forms can be readily applied to other system structures.
\end{abstract}

Index Terms-Battery system, parallel connection, power capability, prediction, state of power.

\section{INTRODUCTION}

Batteries have found promising applications to a variety of areas for energy storage and power supply, from portable electric devices, e.g., cellphones and laptop computers, to large-scale systems, such as electric vehicles (EVs) and power grid energy storage plants. In all these applications, the battery system, composed of cells/modules/packs, has to be charged/discharged within acceptable ranges of current, voltage, power, as well as within available charge/energy capacities. For individual battery cells or packs, these operating limits are specified by manufacturers but only for some given operating scenarios. For instance, the maximum continuous discharging current of the LG ICR $18650 \mathrm{C} 2$ lithium-ion battery cell is $2 \mathrm{C}$ for the ambient temperature between 5 and $45^{\circ} \mathrm{C}$, but it drops drastically to $0.5 \mathrm{C}$ if the temperature falls below $5{ }^{\circ} \mathrm{C}$, where $1 \mathrm{C}$ corresponds to $2700 \mathrm{~mA}$ [1]. These limits, specified only based on special cases, might be too

Manuscript received Month xx, 2xxx; revised Month xx, xxxx; accepted Month x, xxxx. This work was supported by the Swedish Energy Agency under project 44401-1. (Corresponding authors: Torsten Wik and Changfu Zou.)

W. Han, C. Zou, and T. Wik are with the Department of Electrical Engineering, Chalmers University of Technology, Gothenburg 41296, Sweden (weiji.han@chalmers.se, changfu.zou@ chalmers.se, tw@chalmers.se).

Faisal Altaf is with the Volvo Group Trucks Technology, Gothenburg, Sweden (faisal.altaf@volvo.com). conservative or aggressive in practical applications where the working conditions and functional requirements vary in wide ranges, e.g., the acceleration, climbing, or regenerative braking of EVs during summer or winter days. To ensure the safe and efficient operation, the maximum acceptable current or power of the battery system should, therefore, be dynamically updated on the fly according to the real-time system states and operating conditions. This problem is commonly referred to as the prediction or estimation of the battery system's peak charge/discharge power [2], [3], power capability [4]-[7], or, more frequently, state of power (SoP) [8]-[13].

In this paper, the SoP of a battery system during charging (discharging) is defined by the maximum constant current with which the battery system can be continuously charged (discharged) in the following time horizon of concern, i.e., the prediction horizon, without violating any cell-level operating constraints. Once the current-defined SoP has been predicted, the maximum charging or discharging power during this prediction horizon can then be accordingly predicted based on the system's terminal voltage. Clearly, a too conservative prediction of the battery SoP indicates a waste of certain available power capability. On the other hand, a too aggressive SoP prediction could incur a series of progressive issues, such as high power loss, rapid temperature increase, premature termination of charging/discharging, accelerated degradation, and even circuit faults, thermal runaway, and fire hazards. Thus, it is crucially important to accurately predict the SoP of a battery system. Moreover, only based on precise SoP prediction can the controller effectively regulate the system current or power for optimized performance. For instance, the accurate prediction of a battery system's charging SoP can be directly used for the fast charging in battery-powered applications [4], such as smartphones and EVs.

Depending on the application scenarios of battery systems, the number of battery cells involved can vary from one in, e.g., a cellphone, to thousands in, e.g., an EV. The SoP prediction/estimation at the battery cell level has been studied in recent literature [4]-[7], [9]-[11], [13], [14], and a comparative review of on-board SoP prediction for lithiumion batteries in EVs has been presented in [12]. The SoP prediction for an entire multi-battery system, however, has been less investigated to date. The most common method in both industrial practice and academic research is to first predict the SoPs of individual cells in the system, and then infer the battery system's SoP from these cell SoPs. This method is, thus, referred to as the cell-SoP-based method. A typical cell-SoP-based method is the Hybrid Pulse Power 
Characterization Test, introduced for determining the dynamic power capability over the device's usable charge and voltage range, i.e., the vehicle battery pack's SoP [15]. The basic idea of this method is to first predict one battery cell's SoP and then scale it up to the battery pack level through a pre-specified Battery Size Factor. In other words, the battery system's SoP is indeed predicted based on one single cell's SoP. Another cell-SoP-based method was proposed in [8] by applying a comprehensive battery cell model to each cell's SoP estimation. However, when deducing the system SoP from cell SoPs, the dynamic current distribution among parallelconnected battery cells/modules/packs was not considered.

The above cell-SoP-based methods are readily applicable to the SoP prediction of a battery system consisting of only series-connected cells. In such a system, all cells always share the identical current, and, hence, the system SoP in terms of a constant system current is basically the cell SoP of the minimum magnitude [2], [3]. Once any parallel-connected cells are present, the current distribution among them is always varying even given a constant system current during the prediction horizon, as will be illustrated later in Section II. Thus, the system's SoP cannot be exactly derived from associated cells' SoPs because they are defined by constant cell currents during the prediction horizon. To roughly predict the SoP of a battery system with parallel-connected units based on cell SoPs, the dynamic current distribution among parallel cells/modules is either ignored, e.g., simply using a constant sizing factor as in [15], or assumed to be uniform regardless of the heterogeneous cell open circuit voltages (OCVs), charge capacities, or impedances as in [8]. Consequently, the system SoP obtained based on cell SoPs potentially risks overprediction or underprediction, leading to overutilization or underutilization of the battery system accordingly (See the illustration in Section V-E).

In order to accurately predict the SoP of a battery system including parallel-connected units, comprehensive modeling and analysis of the entire battery system's operation, especially the dynamic parallel current distribution, are required. Parallel operation of battery cells has been modeled for performance evaluation [16]-[19] as well as sensitivity analysis to heterogeneous cell resistance [16], [17], [19]-[21], charge capacity [16], [17], [19], [21]-[24], number of parallel cells [18], [23], and system configuration [16]. However, to the best of our knowledge, the complete battery system modeling considering time-varying parallel current distribution has not been applied to the battery system SoP prediction due to significantly increased complexity in system modeling and algorithm design as compared to those commonly used cell-SoP-based methods. To address these critical challenges in the SoP prediction for battery systems with heterogeneous and parallel units, which will be particularly analyzed in Section II, an efficient systemmodel-based SoP prediction method has been first proposed in this paper.

In this work, major contributions have been made in the problem formulation and algorithm design to achieve optimality, high computational efficiency, and wide applicability. Specifically, to pursue the optimal solution, the battery system SoP prediction is formulated as an optimization problem with respect to both the cell/string index and the time instant, instead of only searching the cell/string index hitting the operating limit of concern in the cell-SoP-based method. To solve this problem, a system-model-based algorithm has been developed, and its efficacy and advantage have been verified through tests on a virtual battery system established with experimentally validated battery parameter values. In particular, the computational cost is carefully restricted to facilitate online applications by designing a three-step framework and applying linear models to each short SoP prediction horizon while maintaining optimality and sufficient modeling accuracy. Moreover, the proposed SoP prediction method can be widely applied to various battery connection structures, especially those with parallel-connected units, since the problem formulation and algorithm design are both based on the generic battery model and state-space representation.

\section{Challenges of PREDicting BAtTERy System SoP}

As defined in Section I, both the battery cell-level SoP and system-level SoP are subject to cell-level operating limits typically enforced on the cell current, terminal voltage, state of charge (SoC), etc. The cell SoP is a constant cell current during the prediction horizon, and, thus, can be directly constrained by these cell-level limits. However, because the behaviors of all cells in the system are controlled through a system-level input, the system SoP is defined by a constant system current subject to cell-level constraints. To bridge the gap between system-level input and cell-level system behavior, the cell interconnection needs to be considered.

For a battery system composed of only series-connected cells, the system current and each cell current are identical, which makes it straightforward to predict the system SoP from individual cell SoPs. Once the parallel connection is introduced, the system current is not equal to the current of any individual parallel branch but their sum. Furthermore, the system current is not evenly distributed to parallel-connected cells/modules due to heterogeneous cell SoCs, ages, temperatures, or nonzero parallel connection resistances, and the distribution ratios change over time along with the battery dynamics. While the battery SoC imbalance can be mitigated through external circuitry [25], [26], other factors, such as parameter heterogeneity and battery dynamics, still exist. As a consequence, given a constant system current for a certain time horizon, the constrained cell-level variables, such as the cell current and cell terminal voltage, could evolve in various patterns. For instance, as illustrated in Fig. 1, given that the constant charging current to a battery system is updated every second, it can be observed that during each second the cell's current or terminal voltage could increase, decrease, or exhibit a local minimum or maximum.

Fig. 1 reveals a critical difference in the evolution of constrained cell-level variables between predicting the battery cell SoP and system SoP. In the cell SoP prediction, thanks to the monotonic system evolution under a constant cell current, we only need to check the variable values of concern at the beginning or the end of the prediction horizon. Nevertheless, in the operation of a battery system, these variables might 


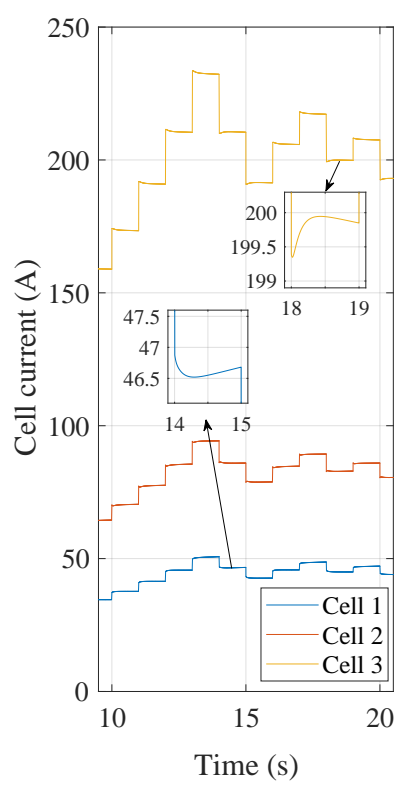

(a)

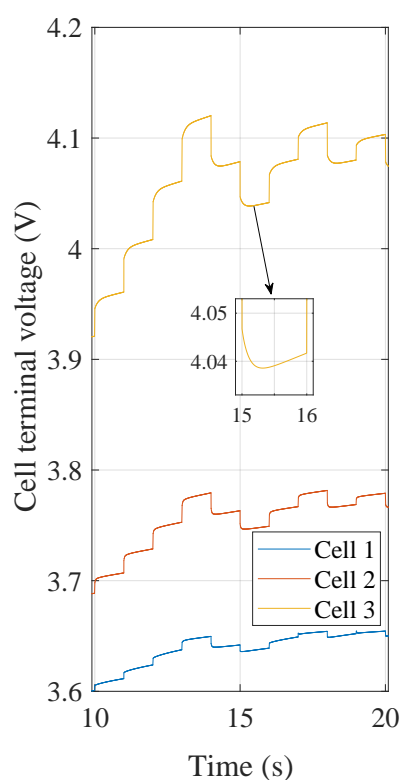

(b)
Fig. 1. Evolution of cell current and terminal voltage in a battery system consisting of three parallel cells given a constant system charging current updated every second. (a) Cell current evolution. (b) Cell terminal voltage evolution.

follow non-monotonic evolution patterns and hit their upper or lower bounds at some time instant during the prediction horizon. Therefore, not only the initial and final time instants but the intermediate period of each prediction horizon also needs to be considered in the system SoP prediction. Such challenges originating from the parallel connection and its influence on the system SoP prediction, however, have never been systematically addressed in the literature. To bridge this research gap, in this work, we will investigate all battery cells' evolution throughout the prediction horizon, and identify the possible maximum or minimum values of these constrained variables along with the corresponding time instants. To do so, the battery system model along with it state-space representation need to be formulated.

\section{MODELING OF A BATTERY PACK SYSTEM WITH PARALLEL CONNECTION}

\section{A. Equivalent circuit model of a battery pack system with parallel-connected units}

Battery systems with parallel-connected units can be set up with different connection structures. To demonstrate the development of the proposed SoP prediction method for such systems, a battery pack system consisting of parallel battery strings is taken as an example, in which each battery string is composed of one battery cell or the same number of seriesconnected cells. Depending on the number and interconnection of in-pack cells/strings, the battery pack system can represent one single cell, one string composed of series-connected cells, parallel-connected cells, as well as parallel-connected strings. When such battery packs are then connected in series to form a larger-scale system, the entire system's SoP still depends on the pack SoP of the lowest magnitude since all packs always share the same current. Thus, all the following analyses and the SoP prediction will be performed on such a battery pack system composed of parallel battery strings.

To analyze the battery system operation, the battery pack system needs to be appropriately modeled, including both individual battery cells and their connection topology. The battery equivalent circuit model (ECM) is deployed here to model each battery cell, and all wires connecting battery cells are characterized by resistors. The battery ECM is widely applied in research studies and industrial applications because its model parameters can be updated online and the currentvoltage behavior can then be predicted with high accuracy but low computational demand. An ECM diagram of the above battery pack system, consisting of $M$ parallel strings and $N$ serial cells per string, is presented in Fig. 2. To simplify the expression, all time indices are omitted throughout this section.

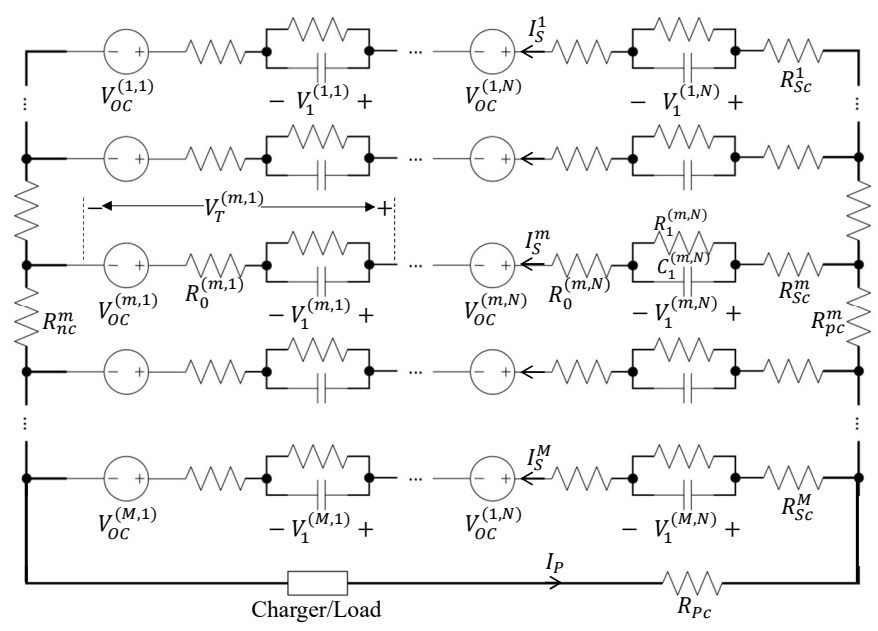

Fig. 2. ECM diagram of a battery pack system comprising $M$ parallel strings with $N$ cells connected in series on each string.

As illustrated in Fig. 2, each battery cell ECM is composed of one voltage source representing its open circuit voltage (OCV), $V_{O C}^{(m, n)}, m \in\{1,2, \ldots, M\}, n \in\{1,2, \ldots, N\}$, one resistor characterizing its internal resistance, $R_{0}^{(m, n)}$, and at least one parallel RC pair to capture the voltage dynamics. In each RC pair, the resistance and capacitance are denoted by $R_{j}^{(m, n)}$ and $C_{j}^{(m, n)}$, respectively, and the corresponding RC pair's voltage is denoted by $V_{j}^{(m, n)}, j=1,2, \ldots, J$, where $J$ is the order of the battery cell ECM. Although the firstorder battery ECM is shown in Fig. 2 for clear illustration, i.e., $J=1$, the ECM with multiple RC pairs will also be considered in the following analysis and prediction. Then, based on Fig. 2, each cell's terminal voltage is

$$
\begin{gathered}
V_{T}^{(m, n)}=V_{O C}^{(m, n)}+I_{S}^{m} R_{0}^{(m, n)}+\sum_{j=1}^{J} V_{j}^{(m, n)}, \\
m \in\{1,2, \ldots, M\}, n \in\{1,2, \ldots, N\},
\end{gathered}
$$

where $I_{S}^{m}$ denotes the current through all cells in the $m$-th string.

To model the wiring among battery cells and the 
charger/load, connection resistors are added in Fig. 2. Specifically, all cell connection resistances on each string are aggregated into a connection resistance denoted by $R_{S c}^{m}, m \in$ $\{1,2, \ldots, M\}$. Besides, between adjacent parallel strings, the connection resistance on the positive and negative terminals are denoted by $R_{p c}^{m}$ and $R_{n c}^{m}$, respectively. In addition, the charger/load is connected to the battery pack system through a wire with its total resistance denoted by $R_{P c}$.

\section{B. State-space representation of serial cells on all strings}

For each battery cell, to facilitate the linear expression of the cell terminal voltage, the cell OCV $V_{O C}^{(m, n)}$ and the RC pair voltage $V_{j}^{(m, n)}, j=1, \ldots, J$, are chosen as state variables. Then the system state vector is defined by

$$
\begin{aligned}
x= & {\left[V_{O C}^{(1,1)}, V_{1}^{(1,1)}, \ldots, V_{J}^{(1,1)}, V_{O C}^{(1,2)}, V_{1}^{(1,2)}, \ldots, V_{J}^{(1,2)},\right.} \\
& \left.\ldots, V_{O C}^{(M, N)}, V_{1}^{(M, N)}, \ldots, V_{J}^{(M, N)}\right]^{T}, x \in \mathbb{R}^{(J+1) M N} .
\end{aligned}
$$

Let $z^{(m, n)}$ denote the cell's SoC, defined by the ratio of its present amount of charge $Q^{(m, n)}$ and its charge capacity $Q_{C}^{(m, n)}$. Given the cell's OCV-SoC curve, influenced by the cell's temperature, charge capacity $Q_{C}^{(m, n)}$, and current direction (charging or discharging), the corresponding cell OCV $V_{O C}^{(m, n)}$ can be determined and the OCV-SoC slope is $k_{V S}^{(m, n)}\left(z^{(m, n)}\right)=\frac{\mathrm{d} V_{O C}^{(m, n)}}{\mathrm{d} z^{(m, n)}}$. Then,

$$
\begin{aligned}
\frac{\mathrm{d} V_{O C}^{(m, n)}}{\mathrm{d} t} & =\frac{\mathrm{d} V_{O C}^{(m, n)}}{\mathrm{d} z^{(m, n)}} \frac{\mathrm{d} z^{(m, n)}}{\mathrm{d} t}=\frac{k_{V S}^{(m, n)}\left(z^{(m, n)}\right)}{Q_{C}^{(m, n)}} \frac{\mathrm{d} Q^{(m, n)}}{\mathrm{d} t} \\
& =\frac{k_{V S}^{(m, n)}\left(z^{(m, n)}\right)}{Q_{C}^{(m, n)}} I_{S}^{m} .
\end{aligned}
$$

Moreover, applying Kirchhoff's current law (KCL) to each RC pair leads to

$$
\begin{aligned}
& \frac{\mathrm{d} V_{j}^{(m, n)}}{\mathrm{d} t}=\frac{1}{C_{j}^{(m, n)}}\left(I_{S}^{m}-\frac{V_{j}^{(m, n)}}{R_{j}^{(m, n)}}\right) \\
& =\frac{-1}{R_{j}^{(m, n)} C_{j}^{(m, n)}} V_{j}^{(m, n)}+\frac{1}{C_{j}^{(m, n)}} I_{S}^{m}, \\
& j=1, \ldots, J, m=1, \ldots, M, n=1, \ldots, N .
\end{aligned}
$$

Denote the string current vector by $I_{S}=\left[I_{S}^{1}, \ldots, I_{S}^{M}\right]^{T}$, $I_{S} \in \mathbb{R}^{M}$. Then, the state-space representation of all battery cells can now be generalized from (2) and (3) as

$$
\dot{x}=A_{S} x+B_{S} I_{S} .
$$

\section{Relation between the string currents and the pack current}

Due to the dynamic current distribution among parallel battery strings, the control input in (4), i.e., all string currents, are interdependent on each other and difficult to be individually assigned. In industrial applications, it is usually the entire battery pack's current, i.e., $I_{P}$ in Fig. 2, that is feasible to be controlled. Thus, the relation between all string currents and the pack current needs to be analyzed.

Applying Kirchhoff's voltage law (KVL) to each loop, composed of two adjacent battery strings along with their connection resistors in Fig. 2, leads to

$$
\begin{aligned}
& \sum_{n=1}^{N} V_{O C}^{(m, n)}+\sum_{n=1}^{N} \sum_{j=1}^{J} V_{j}^{(m, n)}+I_{S}^{m}\left(\sum_{n=1}^{N} R_{0}^{(m, n)}+R_{S c}^{m}\right) \\
& +\sum_{k=1}^{m} I_{S}^{k}\left(R_{p c}^{m}+R_{n c}^{m}\right)-\sum_{n=1}^{N} V_{O C}^{(m+1, n)}-\sum_{n=1}^{N} \sum_{j=1}^{J} V_{j}^{(m+1, n)} \\
& -I_{S}^{m+1}\left(\sum_{n=1}^{N} R_{0}^{(m+1, n)}+R_{S c}^{m+1}\right)=0 \\
& m=1,2, \ldots, M-1 .
\end{aligned}
$$

Additionally, applying KCL to the positive terminal of the battery pack results in

$$
\sum_{m=1}^{M} I_{S}^{m}=I_{P} .
$$

Reorganizing the above $M$ equations in (5) and (6) yields

$$
E x+F I_{S}=G I_{P} .
$$

Thus, string currents can be represented by the pack current,

$$
\begin{aligned}
I_{S} & =C_{I} x+D_{I} I_{P}, \\
\text { where } C_{I} & =-F^{-1} E \text { and } D_{I}=F^{-1} G .
\end{aligned}
$$

It can be seen from (8) that a constant pack current $I_{P}$ leads to time-varying string and cell currents $I_{S}$ because the system state $x$ is involved, which explains the observation in Fig. 1a. This, again, indicates that the exact battery pack SoP cannot be obtained by comparing cell SoPs in the presence of parallel connection, but has to be predicted based on the battery pack model.

\section{State-space representation of the battery pack system}

Finally, based on (4), (8), and (9), the state-space representation for the battery system is formulated as

$$
\begin{aligned}
\dot{x} & =A x+B I_{P}, \\
\text { where } A & =A_{S}-B_{S} F^{-1} E \text { and } B=B_{S} F^{-1} G .
\end{aligned}
$$

Clearly, the input of this system now becomes the pack system's current $I_{P}$, distinguishing the battery pack SoP from the single cell SoP. The matrices $A_{S}, B_{S}, E, F$, and $G$ in (11) can be derived based on (2), (3), (5), and (6), and they will not be detailed here for brevity. Note that $B_{S}$ is statedependent based on the cell OCV-SoC curve, and so are $A$ and $B$. Seemingly linear, (10) is actually a nonlinear system.

In addition, as important variables constrained in the battery pack SoP prediction, the vector of cell terminal voltages, denoted by $V_{T}=\left[V_{T}^{(1,1)}, V_{T}^{(1,2)}, \ldots, V_{T}^{(M, N)}\right]^{T}, V_{T} \in \mathbb{R}^{M N}$, can be derived according to (1) and (8) and viewed as an output of the system (10),

$$
V_{T}=C_{V} x+D_{V} I_{P}
$$

\section{SySteM-MODEL-BASED BATTERY PACK SOP PREDICTION}

To maintain the safe operation of a battery system, each battery cell needs to operate within certain constraints com- 
monly imposed on each cell's current, SoC, terminal voltage, etc. In this section, the battery system SoP considering these constraints will be predicted based on the state-space representation formulated in Section III. For battery connection structures other than the one shown in Fig. 2, the proposed prediction procedure for the battery system SoP is also applicable since the formulated state-space representation and the following analyses are all presented in generic forms.

\section{A. Linear system and solutions}

Denote the present time instant by $t_{0}$, and consider a prediction horizon $\left[t_{0}, t_{0}+\Delta t\right)$ for the SoP prediction/estimation. Then, during this horizon, as long as the pack current is bounded by the pack SoP, none of the operating constraints considered should be violated. Note that the pack SoP needs to be updated frequently to adapt to the time-varying system states and parameter values. For example, an updating frequency of $10 \mathrm{~Hz}$ is typically applied to the battery pack SoP prediction in EVs. Denote the update period of pack SoP prediction by $\Delta t_{u}, \Delta t_{u} \leq \Delta t$. Then, the pack SoP predicted at $t_{0}$ for the following $\Delta t$ horizon will not be updated until $t_{0}+\Delta t_{u}$.

During a sufficiently short prediction horizon $\Delta t$, the battery cell's OCV-SoC slope $k_{V S}^{(m, n)}$ can be approximately viewed as constant. For example, when charging the LG ICR18650 C2 lithium-ion battery cell at its maximum rate of $1 \mathrm{C}$ within a wide $\mathrm{SoC}$ range [5\%,95\%], only up to $0.6 \%$ change in the OCV-SoC slope $k_{V S}^{(m, n)}$ can be observed in one prediction horizon $\Delta t=1 \mathrm{~s}$ [1], [27]. Moreover, while the model parameters in Fig. 2 are influenced by factors such as the cell current, SoC, temperature, and hysteresis, these factors do not change substantially given a constant pack current, e.g., the pack SoP, during a sufficiently short period. Thus, these parameter values can be assumed constant within a short SoP prediction horizon.

As a result, given a short horizon $\Delta t$, the system matrices in (10) can be approximately regarded as constant, and the battery pack system (10) becomes a linear time-invariant (LTI) system. To allow for various operating constraints, the system output is expressed in a generic form,

$$
y(t)=C x(t)+D I_{P} .
$$

For instance, if it is desired to output the string current, set $C=C_{I}$ and $D=D_{I}$ based on (8). For such a generic LTI system based on (10) and (13), the time-domain solutions during the prediction horizon $t \in\left[t_{0}, t_{0}+\Delta t\right)$ are

$$
\begin{aligned}
x(t) & =e^{A\left(t-t_{0}\right)} x\left(t_{0}\right)+K_{A}\left(t-t_{0}\right) B I_{P}, \\
y(t) & =C e^{A\left(t-t_{0}\right)} x\left(t_{0}\right)+\left(C K_{A}\left(t-t_{0}\right) B+D\right) I_{P}, \\
K_{A}(s) & =\int_{0}^{s} e^{A \tau} d \tau .
\end{aligned}
$$

\section{B. Problem formulation of the pack SoP prediction}

Denote the battery pack SoP in terms of the pack current by $I_{P}^{S o P}$. When $I_{P}^{S o P}$ is fed to the system, at least one output entry, denoted by the $q^{*}$-th entry, will reach the output limit $y^{\text {lim }}$ at a certain time instant $t^{*}$ during the prediction horizon, i.e., $\exists q^{*} \in\{1, \ldots, Q\}$ and $t^{*} \in\left[t_{0}, t_{0}+\Delta t\right]$ such that $y_{q^{*}}\left(t^{*}\right)=y^{\text {lim }}$. Here, $Q$ denotes the number of entries in the output vector, e.g., $Q=M$ for outputting the string current vector $I_{S}$ and $Q=M N$ for the cell terminal voltage vector $V_{T}$. Particularly, in the battery cell SoP prediction, $q^{*}=1$ and $t^{*} \in\left\{t_{0}, t_{0}+\Delta t\right\}$ because the cell output evolves monotonically throughout the prediction horizon. Following this, for the SoP prediction of a string of series-connected cells, only $q^{*}$ needs to be searched. To predict the SoP of a battery pack system with parallel cells/strings, however, both $q^{*}$ and $t^{*}$ need to be identified, which makes the problem more complicated than the SoP prediction for individual or series-connected battery cells.

Let the $q$-th output entry $y_{q}, q \in\{1, \ldots, Q\}$, reach its limit $y^{\lim }$ at some time instant $t \in\left[t_{0}, t_{0}+\Delta t\right]$, then by setting $y_{q}(t)=y^{l i m}$ in (15), the corresponding constant pack current during this prediction horizon is

$$
\begin{aligned}
& I_{P}^{S o P}(q, t)=\frac{y^{l i m}-I_{q} C e^{A\left(t-t_{0}\right)} x\left(t_{0}\right)}{I_{q}\left(C K_{A}\left(t-t_{0}\right) B+D\right)}, \\
& q \in\{1,2, \ldots, Q\}, t \in\left[t_{0}, t_{0}+\Delta t\right],
\end{aligned}
$$

where $I_{q}$ is the $q$-th row of the identity matrix of size $Q$, and these system matrices involved, i.e., $A, B, C$, and $D$, are either pre-calculated or updated at $t=t_{0}$.

It is worth noting that, given the output limit $y^{\text {lim }}$ in (17), the corresponding input pack current $I_{P}^{S o P}$ depends not only on the index of the requested output entry, i.e., $q$, but also on the corresponding time instant $t$ when the limit is reached. Different pack currents can be obtained using (17) depending on the specified output entry and its time of hitting the limit. To ensure the safe operation of the battery pack, none of the entries in the output vector is allowed to exceed the limits throughout the prediction horizon. Thus, among all possible pack currents predicted by (17), the one of minimum magnitude is selected as the the battery pack's SoP, i.e.,

$$
\left|I_{P}^{S o P}\right|=\left|I_{P}^{S o P}\left(q^{*}, t^{*}\right)\right|=\min _{\substack{q \in\{1,2, \ldots, Q\} \\ t \in\left[t_{0}, t_{0}+\Delta t\right]}}\left|I_{P}^{S o P}(q, t)\right| .
$$

According to (18), the exact battery pack SoP is impossible to obtain by exhaustive search. Therefore, a computationally efficient algorithm will be designed to solve this problem.

\section{Pack SoP prediction algorithm}

1) Preliminary prediction: Due to the possible nonmonotonic evolution of constrained output variables, such as the string current and cell terminal voltage illustrated in Fig. 1, the maximum or minimum over the prediction horizon might occur at the beginning $t=t_{0}$, at the end $t=t_{0}+\Delta t$, or in between. To generate a preliminary prediction of the pack SoP, the first two special cases are considered, i.e., assuming $t^{*} \in\left\{t_{0}, t_{0}+\Delta t\right\}$. Then, based on (17) and (18), a preliminary pack SoP prediction, denoted by $I_{P}^{p r e}$, can be obtained by

$$
\begin{aligned}
\left|I_{P}^{\text {pre }}\right|=\min \left\{\left|I_{P}^{S o P}\left(1, t_{0}\right)\right|,\left|I_{P}^{S o P}\left(1, t_{0}+\Delta t\right)\right|,\right. \\
\left.\ldots,\left|I_{P}^{S o P}\left(Q, t_{0}\right)\right|,\left|I_{P}^{S o P}\left(Q, t_{0}+\Delta t\right)\right|\right\} .
\end{aligned}
$$


This preliminary prediction serves as an initial guess of the pack SoP, which can be directly searched among $2 Q$ pack currents evaluated using (17). Note that, to lessen the computational burden, the matrix exponential $e^{A\left(t-t_{0}\right)}$ and its integral $K_{A}\left(t-t_{0}\right)$ at $t=t_{0}+\Delta t$ only need to be evaluated once during each update period.

2) Checking feasibility: The feasibility of the preliminary prediction still depends since all intermediate outputs have not been checked yet. Thus, $I_{P}^{p r e}$ is fed to the system (10) and the output values are sampled based on, e.g., (8) or (12). Then, any output violation among all sampling points at $t=t_{0}+s \Delta t_{s}$, $s=0, \ldots, N_{s}$, will be detected, where $\Delta t_{s}=\Delta t / N_{s}$ is the sampling period. If no output exceeds the limit, $I_{P}^{p r e}$ is feasible and we terminate the prediction by setting $I_{P}^{S o P}=I_{P}^{p r e}$. Otherwise, record the set of output entries exceeding their limits, denoted by $\Omega^{e x}$, along with their sampled maximum excesses and corresponding sampling instants.

3) Removing excesses: If $I_{P}^{\text {pre }}$ is infeasible, each output $y_{q \in \Omega^{e x}}$ has at least one peak (valley) between $t_{0}$ and $t_{0}+$ $\Delta t$ violating the limit during charging (discharging). Given a constant pack current and a short-term prediction horizon, e.g., $\Delta t=1 \mathrm{~s}$, the output variables of concern, such as the cell current or terminal voltage, do not oscillate. Thus, each output $y_{q \in \Omega^{e x}}$ is assumed to have only one peak or valley during the prediction horizon. To remove the excesses in these output entries, let each output $y_{q \in \Omega^{e x}}$ reach the limit and its own peak (valley) simultaneously during the prediction horizon of the charging (discharging) process, which gives the conditions

$$
y_{q}(t)=y^{l i m}, \dot{y}_{q}(t)=0, q \in \Omega^{e x} .
$$

According to (15), the former results in the $I_{P}$ derived in (17), and the latter leads to

$$
\dot{y}_{q}(t)=I_{q} C e^{A\left(t-t_{0}\right)}\left(A x\left(t_{0}\right)+B I_{P}\right)=0 .
$$

Given any $q \in \Omega^{e x}$, substituting the $I_{P}$ in (20) with the one obtained by (17) results in an equation in time $t$ only, i.e.,

$$
I_{q} C e^{A\left(t-t_{0}\right)}\left(A x_{0}+B \frac{y^{l i m}-I_{q} C e^{A\left(t-t_{0}\right)} x\left(t_{0}\right)}{I_{q}\left(C K_{A}\left(t-t_{0}\right) B+D\right)}\right)=0 .
$$

For each $q \in \Omega^{e x}$, the time instant, at which the $q$-th output is maximized or minimized and hits the limit, can be obtained by solving (21), and it is denoted by $t_{q}^{o p t}$.

To solve (21) for the $t_{q}^{\text {opt }} \in\left(t_{0}, t_{0}+\Delta t\right)$, Newton's method with quadratic convergence rate is deployed. Specifically, the left-hand-side in (21) is viewed as $f(t)$ and, accordingly, $\dot{f}(t)$ can be derived using $\frac{\mathrm{d} e^{A\left(t-t_{0}\right)}}{\mathrm{d} t}=A e^{A\left(t-t_{0}\right)}$ and $\dot{K}_{A}\left(t-t_{0}\right)=$ $e^{A\left(t-t_{0}\right)}$ according to (16). For each output $q \in \Omega^{e x}$, the recorded sampling time instant corresponding to its sampled maximum excess is used as the initial solution $t(0)$. Then, update the solution within $\left[t_{0}, t_{0}+\Delta t\right]$ following

$$
t(k+1)=\min \left\{\max \left\{t_{0}, t(k)-\frac{f(t(k))}{\dot{f}(t(k))}\right\}, t_{0}+\Delta t\right\},
$$

which is recursively applied until the convergence to $t_{q}^{o p t}$.

Based on the $t_{q}^{o p t}$ predicted for each output with excess, the corresponding pack current $I_{P}^{S o P}\left(q, t_{q}^{o p t}\right), q \in \Omega^{e x}$, can be calculated by (17). Finally, the battery pack's SoP is predicted by

$$
\left|I_{P}^{S o P}\right|=\min _{q \in \Omega^{e x}}\left|I_{P}^{S o P}\left(q, t_{q}^{o p t}\right)\right| .
$$

\section{Pack SoP prediction considering multiple operating limits}

The proposed algorithm, derived based on generic system equation (10) and output equation (13), is applicable to accommodate various operating constraints of battery cells. For individual battery cells, the charging/discharging current needs to be strictly limited since a too large current can cause multiple issues, such as high power loss and temperature, fast degradation, and even fire hazard [7], [12]. For interconnected cells, e.g., the system modeled in Fig. 2, because all cells connected in series on each string always share identical current, the cell current limit is also enforced on the string current. To predict the pack SoP considering cell/string current limits, the output equation (8) should be deployed in the proposed algorithm.

Moreover, as an important indicator of battery operating status and circuit faults, the cell terminal voltage also needs to be constrained. To predict the battery pack SoP considering such voltage limits, the proposed algorithm based on the output equation (12) can be directly employed. When multiple operating limits are imposed, the pack SoP for each limit can be individually predicted, and the prediction with the lowest magnitude is then selected as the pack SoP for all these limits.

In addition, the upper and lower limits of the battery cell SoC, commonly imposed to avoid overutilizing any battery cell in the pack, have to be treated slightly differently in the pack SoP prediction, since the evolution of the cell OCV-SoC curve's slope $k_{V S}^{(m, n)}$ from present cell SoC to its specified limit needs to be involved. As introduced in Subsection IV-A, the proposed algorithm is derived for a short prediction horizon during which $k_{V S}^{(m, n)}$ can be assumed constant. Thus, only when the present cell $\mathrm{SoC}$ is sufficiently close to its limit can the prediction equation (17) be applied. Therefore, instead of using (19), the cell-SoC-limited pack SoP is preliminarily predicted based on the SoP predictions for other operating limits, e.g., the cell current and terminal voltage limits, and then followed by a feasibility check. If any cell SoC is detected to violate its limit during the prediction horizon, indicating that it already gets very close to the limit at the beginning of this horizon, the proposed procedure for removing the excess becomes applicable. To facilitate the feasibility checking and excess removal, the following output matrices are derived.

To study the cell SoC evolution in a battery system including parallel connections, as compared to Coloumb Counting through the integral of time-varying cell current, it is computationally more efficient to alternatively track the cell OCV since it can be directly extracted from the state vector $x$. Therefore, denote the cell OCV vector by $V_{O C}=$ $\left[V_{O C}^{(1,1)}, V_{O C}^{(1,2)}, \ldots, V_{O C}^{(M, N)}\right]^{T}, V_{O C} \in \mathbb{R}^{M N}$, and express it as the system output

$$
V_{O C}=C_{O C} x+D_{O C} I_{P}
$$

Clearly, $C_{O C}$ is an $M N \times(J+1) M N$ matrix with zero entry entries except $C_{O C}^{(i,(J+1)(i-1)+1)}=1, i \in\{1,2, \ldots, M N\}$, and $D_{O C}$ is an $M N \times 1$ zero vector. 
To wrap up, a flow chart is sketched in Fig. 3 to illustrate the proposed system-model-based SoP prediction algorithm, in which $I_{P, I_{S}}^{S o P}, I_{P, V_{T}}^{S o P}$, and $I_{P, V_{O C}}^{S o P}$ denote the pack SoPs considering the operating limits enforced on the cell/string current $I_{S}$, cell terminal voltage $V_{T}$, and cell OCV $V_{O C}$, respectively.

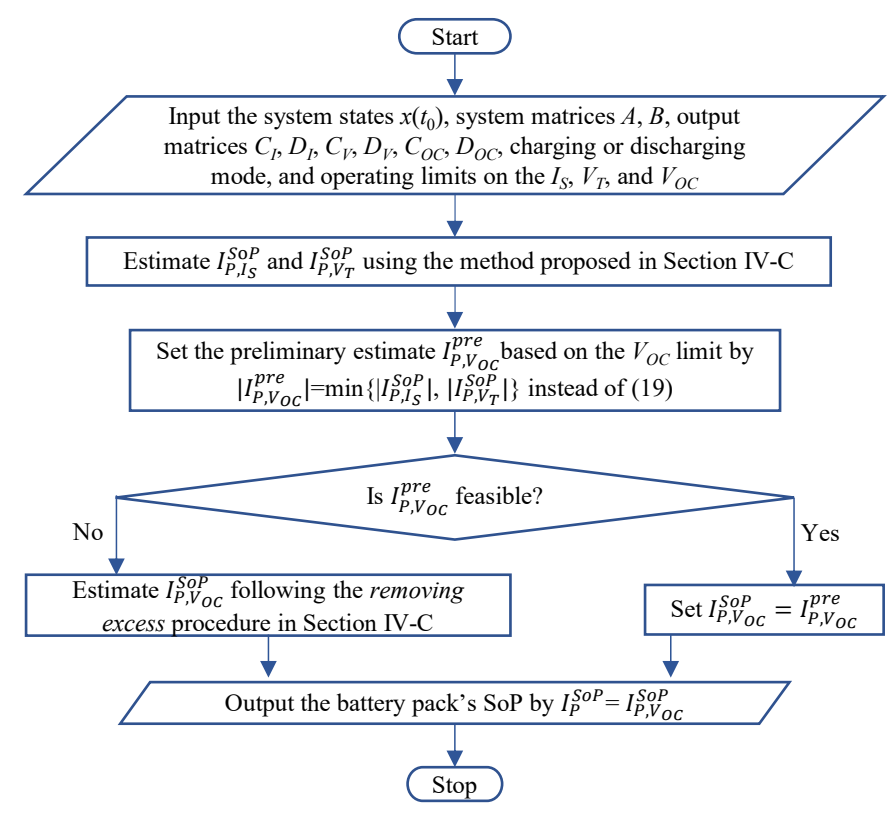

Fig. 3. A flowchart of the proposed system-model-based battery pack SoP prediction algorithm.

\section{TESTS AND ANALYSiS}

It is worth noting that, the predicted charging and discharging SoPs are aimed at indicating the bounds of battery system current/power, and, hence, they are not always implemented during practical operation. Then, to evaluate the performance of the proposed battery system SoP prediction algorithm in Section IV, a common practice in the literature is using modelbased simulation tests, e.g., [4], [10]. Moreover, for the shorthorizon SoP prediction, the model parameter values need to be updated very quickly [28]. Thus, in this work, a secondorder lithium-ion battery ECM with parameter values looked up from tables identified for commercial EV applications is deployed to construct the battery system illustrated in Fig. 2.

\section{A. Identification of look-up tables for battery ECM parameters}

The battery parameter look-up tables were prepared for the applied battery cell based on various battery charging and discharging tests under different current rates, temperatures, and cell SoCs. To do so, a testbed was set up as shown in Fig. 4, where some battery cells were operating in the chamber for constant ambient temperature and others were put on the shelf with room temperature. All cells were charged or discharged with various current rates through the battery tester. While detailed modeling parameter values are omitted due to commercial confidentiality, their dependencies on various factors are presented in Table I. For instance, the battery cell's charge capacity and temperature can affect all cell modeling parameters as well as the cell's OCV-SoC curve. Moreover, the cell's RC-pair parameters, $R_{j}$ and $C_{j}, j=1,2$, are sensitive to the cell's real-time current, and the cell's OCV-SoC curves during charging and discharging are different even given the same cell capacity and temperature.

According to such parameter dependencies, the testing procedure for each cell is designed as follows. (i) Given a battery cell with a pre-estimated charge capacity, specify its temperature through a temperature chamber and its current profile by a battery tester. (ii) The cell was kept in the chamber and charged/discharged following the current profile. During this process, the battery's terminal voltage and surface temperature were sampled and saved in time series. (iii) Based on the battery ECM, specified cell current profile, and collected measurements, parameter values at various cell SoCs were identified using a constrained least squares method, implemented by the MatLab nonlinear optimization toolbox. Following steps (i) to (iii), battery ECM parameters at various cell SoCs for other temperatures and cell capacities were also identified.

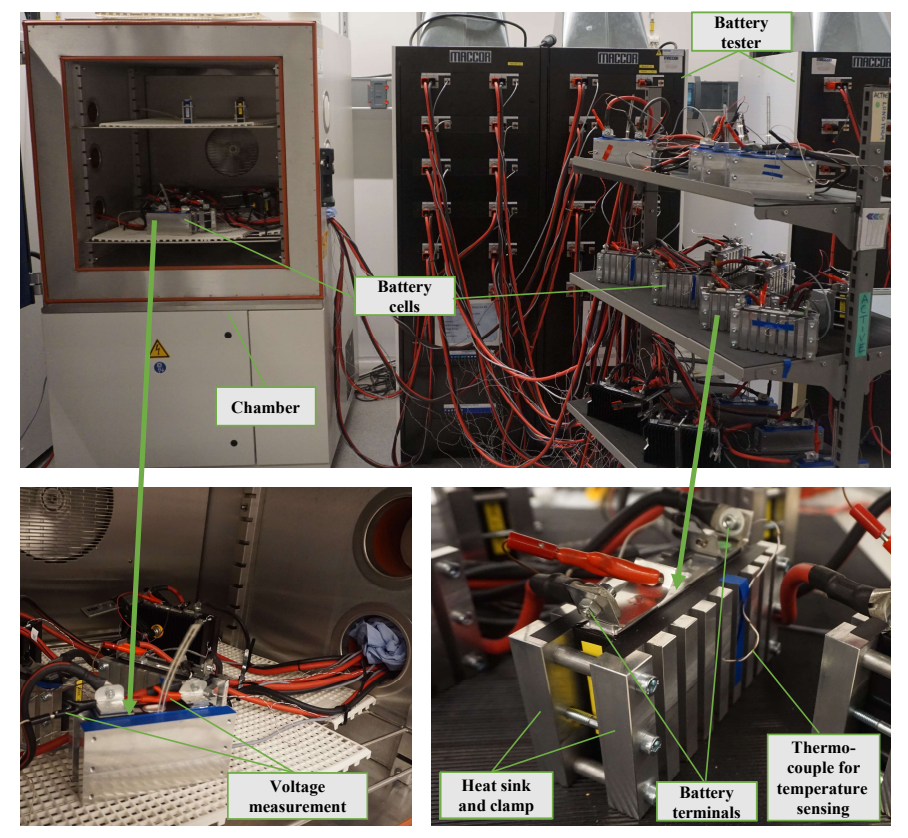

Fig. 4. Testbed for identifying the second-order ECM parameters of battery cells.

TABLE I

DEPENDENCE OF BATTERY CELL PARAMETERS ON VARIOUS FACTORS.

\begin{tabular}{c|c|c|c|c|c}
\hline \hline & \multicolumn{5}{|c}{ Cell-level factors } \\
\hline Cell parameters & Capacity & SoC & Temperature & Current & Hysteresis \\
\hline$R_{0}$ & $\checkmark$ & $\checkmark$ & $\checkmark$ & & \\
$R_{1}, C_{1}, R_{2}, C_{2}$ & $\checkmark$ & $\checkmark$ & $\checkmark$ & $\checkmark$ & \\
$\mathrm{OCV}-\mathrm{SoC}$ curve & $\checkmark$ & & $\checkmark$ & & $\checkmark$ \\
\hline \hline
\end{tabular}

The modeling error of battery cell terminal voltage in terms of mean absolute percentage error (MAPE) has been found 
to be less than $1 \%$ in extensive tests under various relevant scenarios. For instance, as shown in Fig. 5, given the cell current profile at $23^{\circ} \mathrm{C}$ for a new battery cell of full charge capacity (marked by the dashed curve), the measured battery cell terminal voltages are compared with those simulated voltages using the identified battery ECM parameter values. It can be seen that the simulated voltage curve can well match the measured one except for those low-voltage periods. Specifically, the MAPE of all simulated voltages is $0.31 \%$ and the MAPE is $0.16 \%$ for test periods during which the cell SoC stays above 0.1. In EV applications, a typical battery SoC operating window is $[0.15,0.85]$ to avoid issues at very low and high $\mathrm{SoC}$ levels, such as higher aging rates and lower voltage accuracy. Therefore, the battery pack, constructed based on this battery ECM and pre-identified parameter lookup tables, is a high-fidelity virtual battery system for practical applications.

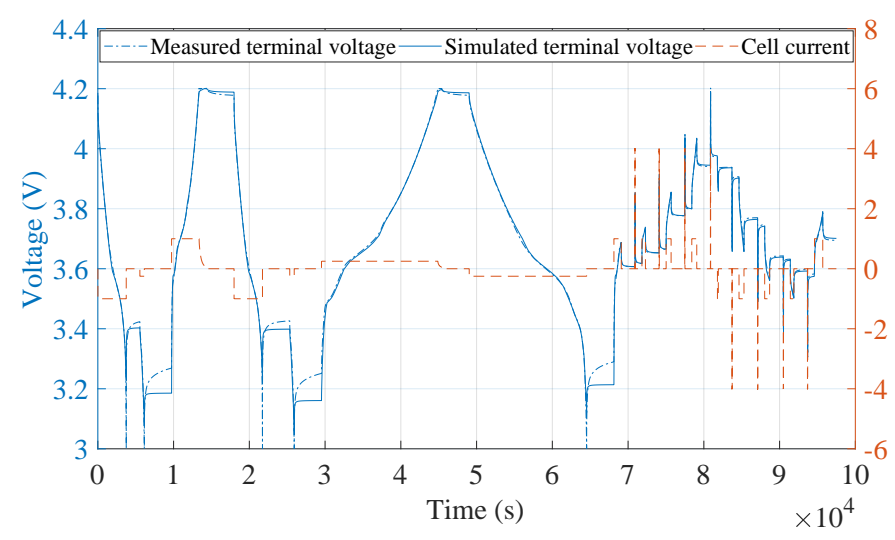

Fig. 5. Comparison of the measured and simulated battery terminal voltages under the given cell current profile at $23{ }^{\circ} \mathrm{C}$ (marked by the dashed curve).

\section{B. Test settings}

Throughout this section, a battery pack system composed of $M=3$ parallel-connected strings and $N=4$ series-connected cells per string will be tested for illustration. In each battery cell model, there are $J=2 \mathrm{RC}$ pairs. Assume all cells are new and the temperature is set to $23{ }^{\circ} \mathrm{C}$. All cell parameters are determined according to the same set of parameter lookup tables of the verified model. Given unbalanced initial cell SoCs and nonzero connection resistances on strings and between strings as shown in Fig. 2, uneven string current distribution can be observed in the following tests. Since the internal resistances of these batteries are quite small and even comparable to the connection resistances assigned in the following tests, the string currents differ from each other significantly for clear illustration.

Note that, as shown in Fig. 6, when predicting the system $\mathrm{SoP}$ at the beginning of each prediction horizon, currentdependent cell parameters, i.e., $R_{1}, C_{1}, R_{2}$, and $C_{2}$ according to Table I, are initially updated based on the predicted cell currents at the end of the last horizon in response to the previous pack SoP prediction. Once an SoP prediction for the present horizon is obtained, the current-dependent cell parameters need to be updated accordingly and the SoP also needs to be predicted again. Such an iterative updating procedure continues until the absolute difference between the two latest SoP predictions is less than a pre-specified threshold, e.g., 0.1 A as used in the tests presented here. Based on the final pack SoP prediction and the requested pack current, the applied pack current is determined. Then, cell parameters need to be updated again to simulate the system operation according to the applied pack current and the resulting string/cell currents.

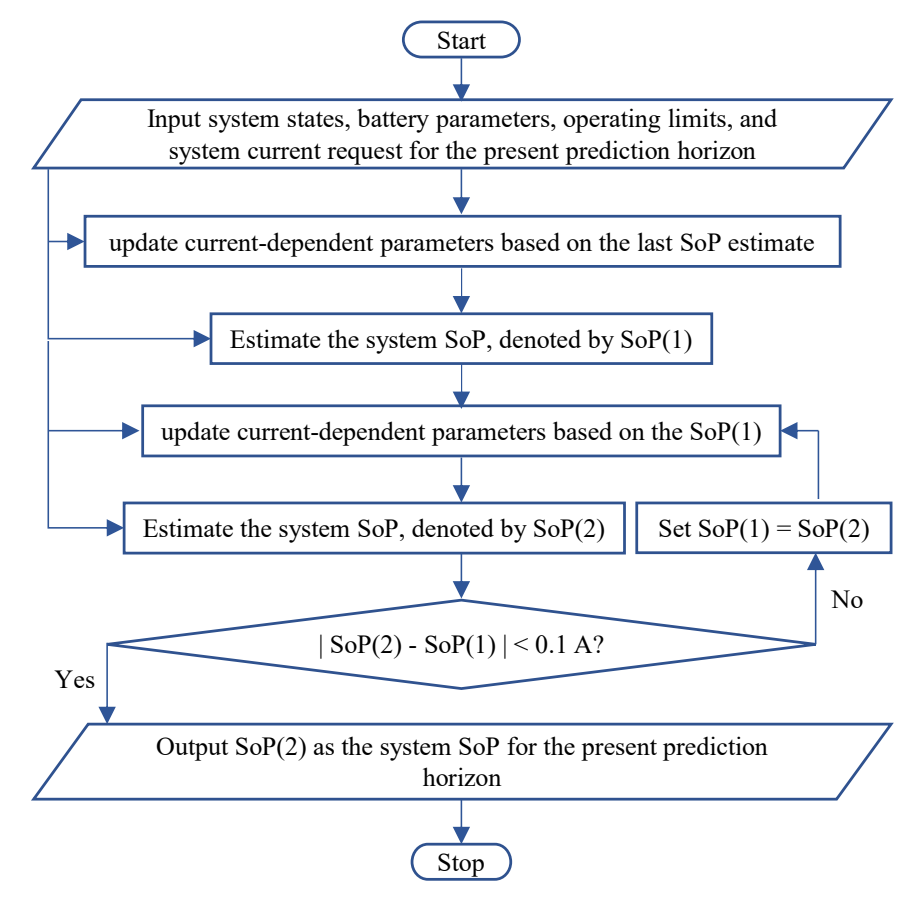

Fig. 6. A flowchart of the iterative update of current-dependent parameters and system SoP prediction at the beginning of each SoP prediction horizon.

\section{Efficacy of the proposed SoP prediction algorithm}

Based on the battery system SoP definition, if the predicted system SoP is applied at the beginning and not updated until the end of the prediction horizon, i.e., $I_{P}(t)=I_{P}^{S o P}, t_{0} \leq$ $t \leq t_{0}+\Delta t, \Delta t_{u}=\Delta t$, some operating limit will be reached but no limits should be exceeded. This will be illustrated in Fig. 7.

Given slightly unbalanced initial cell SoCs within the commonly applied middle-SoC range in practical applications, e.g., 0.5 to $0.55, \Delta t=\Delta t_{u}=1 \mathrm{~s}$, and the upper limits of cell charging current and cell terminal voltage, the pack SoP is predicted using the proposed algorithm in Section IV and applied to the virtual battery system established in Section $\mathrm{V}$-A. The resulting current and voltage evolutions of battery cells during the initial operation period of $50 \mathrm{~s}$ are shown in Fig. 7. Specifically, during the first stage from $0 \mathrm{~s}$ to $22 \mathrm{~s}$, the current of String 3, $I_{S 3}$ (marked by the purple solid curve in Fig. 7 (a)), can reach the upper limit of cell currents, $I_{S}^{u b}$ (marked by the red dashed line). Then, during the remaining operation, the highest cell terminal voltage can reach the upper 


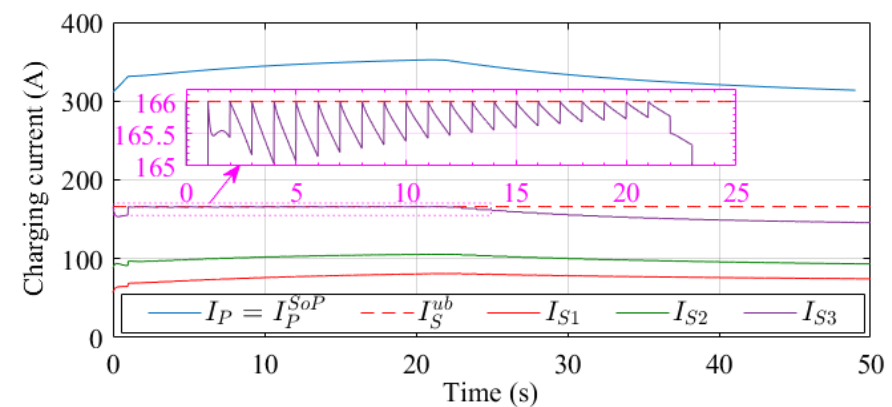

(a)

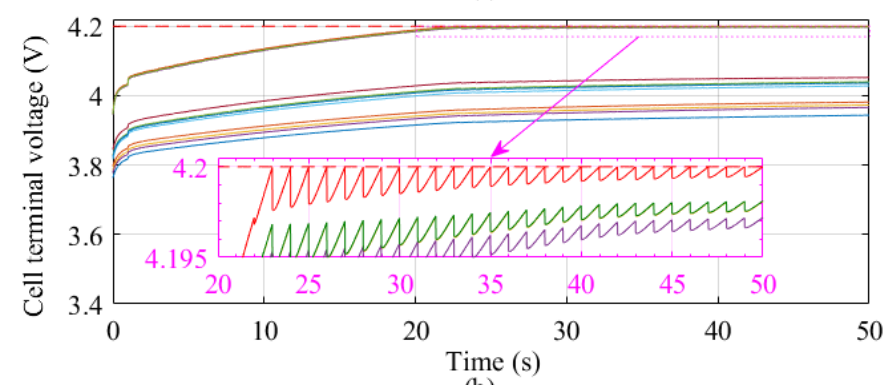

(b)

Fig. 7. Evolutions of constrained variables when applying the predicted pack SoP by the proposed algorithm in Subsection IV throughout each prediction horizon. (a) String current evolution. (b) Cell terminal voltage evolution.

limit as shown in Fig. 7 (b). Thus, throughout this test period, no operating limits are violated, demonstrating the efficacy of the proposed SoP prediction algorithm.

\section{Local maximum or minimum during system evolution}

As pointed out in Section II and shown in Fig. 1, in a battery pack consisting of parallel-connected strings/cells, the system behavior might evolve in various patterns during each prediction horizon. In the proposed prediction algorithm, the preliminary pack SoP $I_{P}^{p r e}$ is predicted only considering the initial and final time instants of the prediction horizon, while the pack SoP $I_{P}^{S o P}$ is predicted based on the entire prediction horizon. To compare these two predictions and their resultant system behaviors, the above system used for illustrating the efficacy of the proposed algorithm is tested again but with a higher updating frequency, $\Delta t_{u}=\Delta t / 2=0.5 \mathrm{~s}$. The preliminary pack SoP $I_{P}^{p r e}$, predicted pack SoP $I_{P}^{S o P}$, and their resultant cell/string current evolutions are compared in Fig. 8.

As can be seen from Fig. 8a, the predicted $I_{P}^{\text {pre }}>I_{P}^{S o P}$ at $t=1 \mathrm{~s}$ and $t=1.5 \mathrm{~s}$, which indicates that at least one string current reaches the upper limit at a certain time instant between the initial and final time instants of the corresponding prediction horizons. As a consequence, when $I_{P}=I_{P}^{p r e}$ is actually applied during these horizons, the string current upper limit is expected to be violated. This is confirmed in Fig. 8b, where we can observe a current excess up to about $0.2 \mathrm{~A}$ during the horizon [1 s, $2 \mathrm{~s}$ ) as well as a smaller excess at the beginning of the following horizon [1.5 s, $2.5 \mathrm{~s}$ ).

Alternatively, when the predicted $I_{P}^{S o P}$ is applied, i.e., $I_{P}=$ $I_{P}^{S o P}$. The resultant string currents, shown in Fig. 8c, all fall within the acceptable operating zone as expected. Moreover,
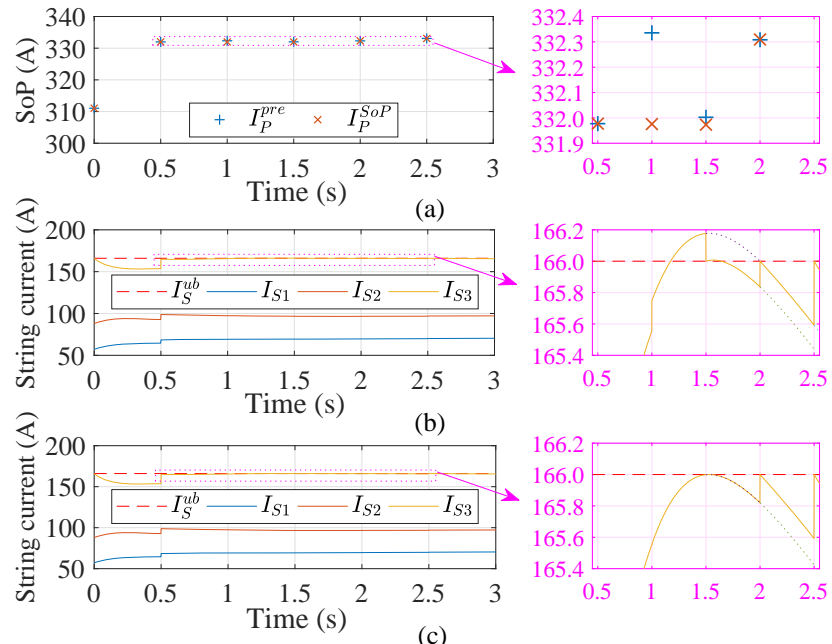

(c)

Fig. 8. (a) Comparison of the preliminary pack SoP $I_{P}^{\text {pre }}$ and the pack SoP $I_{P}^{S o P}$ predicted using the proposed algorithm. (b) String current evolution when applying $I_{P}=I_{P}^{\text {pre }}$ during each update period. (c) String current evolution when applying $I_{P}=I_{P}^{S o P}$ during each update period.

in some discharging tests, it can also be observed that some cell's terminal voltage has a local minimum located neither at the beginning nor at the end of one prediction horizon, and such a special case can also be successfully handled by the proposed SoP prediction algorithm.

\section{E. Comparison with the cell-SoP-based method}

In the commonly applied cell-SoP-based battery system SoP prediction method, battery cells are modeled individually, independent of each other. In the proposed system-modelbased method, in addition to modeling battery cells, their interconnection, especially the dynamic parallel current distribution, is also taken into account. The pack SoP predicted using these two methods, denoted by $I_{P}^{\text {c.SoP }}$ and $I_{P}^{\text {s.model }}$, respectively, along with their corresponding system behaviors are compared in Fig. 9. For both methods, a constant pack discharging current is requested, i.e., $I_{P}^{r e q}=-700 \mathrm{~A}, \Delta t_{u}=\Delta t$ $=1 \mathrm{~s}$, and the same operating limits on the cell/string current, cell terminal voltage, and cell SoC or OCV are imposed.

As illustrated in Fig. 9a, if the pack SoP is predicted by the proposed system-model-based method, i.e., $I_{P}^{S o P}=I_{P}^{\text {s.model }}$, the pack current actually applied is determined by $\left|I_{P}^{a p p}\right|=$ $\min \left\{\left|I_{P}^{r e q}\right|,\left|I_{P}^{\text {s.model }}\right|\right\}$. As a result, no operating limits are violated during the simulation. One of the string currents approaches its lower limit $I_{S}^{l b}$ during the first six seconds, as shown in Fig. 9c, and then some cell's terminal voltage approaches its lower limit $V_{T}^{l b}$ during the remaining horizons, as illustrated in Fig. 9e.

On the other hand, if the battery system SoP is predicted based on cell SoPs, i.e., $I_{P}^{S o P}=I_{P}^{c . S o P}$, the requested pack current $I_{P}^{r e q}$ always has a smaller magnitude than the predicted pack SoP $I_{P}^{c . S o P}$ as shown in Fig. 9b. Thus, the applied pack current is $I_{P}^{a p p}=I_{P}^{r e q}$. Consequently, both cell/string current limit $I_{S}^{l b}$ and the cell terminal voltage $V_{T}^{l b}$ are violated during 


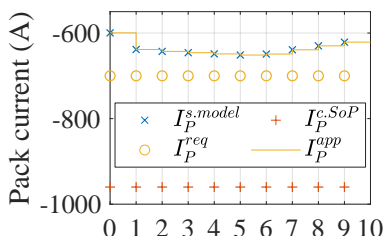

(a)

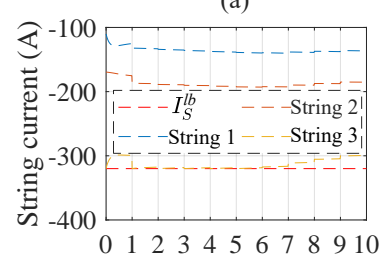

(c)

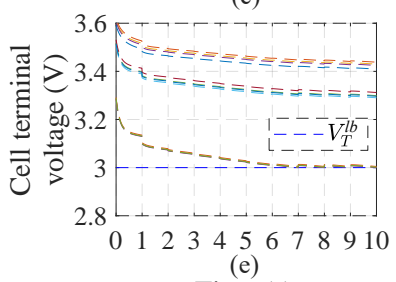

Time (s)

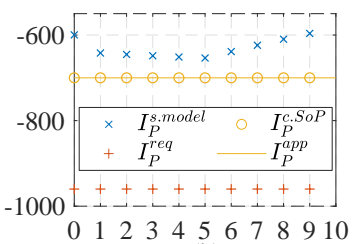

(b)

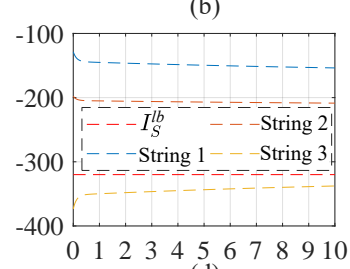

(d)

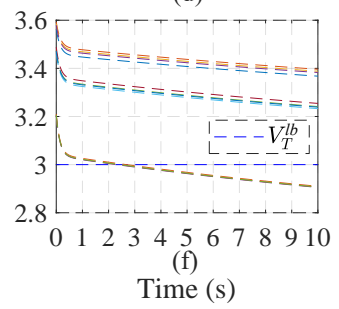

Fig. 9. Comparison of the applied pack current along with the resulting string currents and cell terminal voltages using different SoP prediction methods. $I_{P}^{S o P}=I_{P}^{\text {s.model }}$ for (a), (c), and (e), and $I_{P}^{S o P}=I_{P}^{c . S o P}$ for (b), (d), and (f).

certain periods as illustrated in Fig. 9d and Fig. 9f, indicating that the $I_{P}^{c . S o P}$ is too aggressive to maintain the safe operation. In practice, a predefined constant derating factor $\alpha \in(0,1)$ can be multiplied to $I_{P}^{c . S o P}$ to alleviate the violations. To cover various circumstances, a very conservative factor needs to be selected, indicating that some available power capability might be wasted during certain moderate scenarios. Therefore, the proposed method outperforms the cell-SoP-based method for providing a more accurate and reliable SoP prediction.

\section{CONCLUSIONS AND FUTURE WORK}

State of Power (SoP) has been investigated for battery systems with parallel-connected units. The SoP prediction for such systems is considerably more complicated than that for individual cells because the cell interconnections cause transient current distribution among parallel branches even with constant system current. In addition, different from short-horizon SoP prediction for individual cells, the largest magnitudes of cell currents and voltages may occur any time instant within the SoP prediction horizon.

Consequently, the system SoP prediction method based on individual cell SoPs may therefore lead to unnecessarily conservative predictions or predictions resulting in violation of limits on voltage and current. To bridge this research gap, we have derived and proposed an analytical system-modelbased prediction method, and its efficacy and advantages have been demonstrated through tests on a system model for an electric vehicle application, based on an experimentally validated battery cell model.

The method has been developed based on linear state-space modeling, and, hence, the prediction horizon needs to be restricted for the linearity assumption to be valid. In future work, longer prediction horizons considering the thermal effect and the sensitivity of the battery system SoP to various factors in Table I will be further investigated.

\section{REFERENCES}

[1] B. T. Kim. (2010, Apr.) Product description: ICR18650 C2 $2800 \mathrm{mAh}$. [Online]. Available: http://www.batteryspace.com/prodspecs/5702_5.pdf

[2] B. Jiang, H. Dai, X. Wei, L. Zhu, and Z. Sun, "Online reliable peak charge/discharge power estimation of series-connected lithium-ion battery packs," Energies, vol. 10, no. 3, 2017.

[3] Z. Zhou, Y. Kang, Y. Shang, N. Cui, C. Zhang, and B. Duan, "Peak power prediction for series-connected LiNCM battery pack based on representative cells," J. Cleaner Production, vol. 230, pp. 1061-1073, 2019.

[4] C. Zou, A. Klintberg, Z. Wei, B. Fridholm, T. Wik, and B. Egardt, "Power capability prediction for lithium-ion batteries using economic nonlinear model predictive control," J. Power Sources, vol. 396, pp. 580-589, 2018.

[5] B. Fridholm, T. Wik, H. Kuusisto, and A. Klintberg, "Estimating power capability of aged lithium-ion batteries in presence of communication delays," J. Power Sources, vol. 383, pp. 24-33, 2018.

[6] S. Lee and J. Kim, "Power capability analysis of lithium battery and supercapacitor by pulse duration," Electron., vol. 8, no. 12, Nov. 2019.

[7] X. Tang, Y. Wang, K. Yao, Z. He, and F. Gao, "Model migration based battery power capability evaluation considering uncertainties of temperature and aging," J. Power Sources, vol. 440, p. 227141, 2019.

[8] G. L. Plett, "High-performance battery-pack power estimation using a dynamic cell model," IEEE Trans. Veh. Technol., vol. 53, no. 5, pp. 1586-1593, Sep. 2004.

[9] F. Christian, W. Wladislaw, B. Ziou, and S. Uwe, "Adaptive on-line state-of-available-power prediction of lithium-ion batteries," J. Power Electron., vol. 13, no. 4, pp. 516-527, July 2013.

[10] T. Wik, B. Fridholm, and H. Kuusisto, "Implementation and robustness of an analytically based battery state of power," J. Power Sources, vol. 287, pp. 448-457, 2015.

[11] X. Lai, L. He, S. Wang, L. Zhou, Y. Zhang, T. Sun, and Y. Zheng, "Coestimation of state of charge and state of power for lithium-ion batteries based on fractional variable-order model," J. Cleaner Production, vol. 255, p. 120203, May 2020.

[12] A. Farmann and D. U. Sauer, "A comprehensive review of on-board state-of-available-power prediction techniques for lithium-ion batteries in electric vehicles," J. Power Sources, vol. 329, pp. 123-137, 2016.

[13] X. Hu, H. Jiang, F. Feng, and B. Liu, "An enhanced multi-state estimation hierarchy for advanced lithium-ion battery management," Appl. Energy, vol. 257, p. 114019, 2020.

[14] Y. Wang, J. Tian, Z. Sun, L. Wang, R. Xu, M. Li, and Z. Chen, "A comprehensive review of battery modeling and state estimation approaches for advanced battery management systems," Renewable Sustain. Energy Rev., vol. 131, p. 110015, 2020.

[15] G. Hunt. (2001, Feb.) PNGV battery test manual. [Online]. Available: https://avt.inl.gov/sites/default/files/pdf/battery/pngv_manual_rev3b.pdf

[16] L. Crotta, "Heterogeneity and sensitivity analysis for multi-battery control," M.S. thesis, Politecnico di Milano, Milano MI, Italy, Dec. 2018.

[17] T. Bruen and J. Marco, "Modelling and experimental evaluation of parallel connected lithium ion cells for an electric vehicle battery system," J. power sources, vol. 310, pp. 91-101, 2016.

[18] F. Chang, F. Roemer, M. Baumann, and M. Lienkamp, "Modelling and evaluation of battery packs with different numbers of paralleled cells," World Elect. Veh. J., vol. 9, no. 1, 2018.

[19] M. J. Brand, M. H. Hofmann, M. Steinhardt, S. F. Schuster, and A. Jossen, "Current distribution within parallel-connected battery cells," J. Power Sources, vol. 334, pp. 202-212, 2016.

[20] R. Gogoana, M. B. Pinson, M. Z. Bazant, and S. E. Sarma, "Internal resistance matching for parallel-connected lithium-ion cells and impacts on battery pack cycle life," J. Power Sources, vol. 252, pp. 8-13, 2014.

[21] S. Miyatake, Y. Susuki, T. Hikihara, S. Itoh, and K. Tanaka, "Discharge characteristics of multicell lithium-ion battery with nonuniform cells," J. Power Sources, vol. 241, pp. 736-743, 2013.

[22] C. Pastor-Fernández, T. Bruen, W. Widanage, M. Gama-Valdez, and J. Marco, "A study of cell-to-cell interactions and degradation in parallel strings: implications for the battery management system," J. Power Sources, vol. 329, pp. 574-585, 2016. 
[23] A. Fill, S. Koch, A. Pott, and K.-P. Birke, "Current distribution of parallel-connected cells in dependence of cell resistance, capacity and number of parallel cells," J. Power Sources, vol. 407, pp. 147-152, 2018.

[24] M.-S. Wu, C.-Y. Lin, Y.-Y. Wang, C.-C. Wan, and C. Yang, "Numerical simulation for the discharge behaviors of batteries in series and/or parallel-connected battery pack," Electrochimica acta, vol. 52, no. 3, pp. 1349-1357, 2006.

[25] X. Tang, C. Zou, T. Wik, K. Yao, Y. Xia, Y. Wang, D. Yang, and F. Gao, "Run-to-run control for active balancing of lithium iron phosphate battery packs," IEEE Trans. Power Electron., vol. 35, no. 2, pp. 1499$1512,2020$.

[26] W. Han, C. Zou, C. Zhou, and L. Zhang, "Estimation of cell SOC evolution and system performance in module-based battery charge equalization systems," IEEE Trans. Smart Grid, vol. 10, no. 5, pp. 47174728 , Sept. 2019.

[27] LG $18650 \quad \mathrm{C} 2 \quad 2800 \mathrm{mAh}$ test data. (Accessed on Sep. 13th, 2019). [Online]. Available: https://lygteinfo.dk/review/batteries2012/Common18650comparator.php

[28] M. Kim, K. Kim, and S. Han, "Reliable online parameter identification of li-ion batteries in battery management systems using the condition number of the error covariance matrix," IEEE Access, vol. 8, pp. 189 106-189 114, 2020.

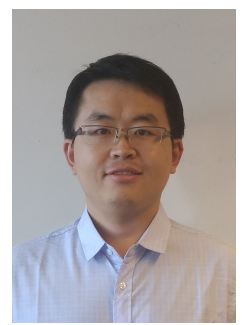

Weiji Han (Member, IEEE) received the B.E. and M.E. degrees from the Department of Electrical Engineering, Shandong University, China, in 2009 and 2012, respectively, and the second M.E. degree and the Ph.D. degree in Electrical Engineering from the Department of Electrical and Computer Engineering, University of Connecticut, Storrs, CT, USA, in 2015 and 2018, respectively.

$\mathrm{He}$ is currently a Researcher in the Department of Electrical Engineering, Chalmers University of Technology, Gothenburg, Sweden. His research interests include modeling, analysis, and control of battery systems with applications to electric vehicles and power grid energy storage. Besides, he also has research experiences in the power system, photovoltaic system, and manufacturing system.

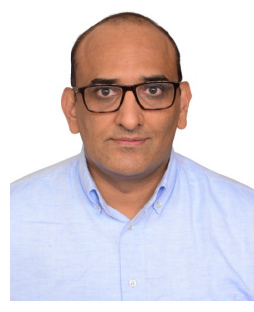

Faisal Altaf (Member, IEEE) received the B.Sc. degree in Mechatronics Engineering from National University of Sciences and Technology (NUST), Islamabad, Pakistan, the M.Sc. degree in Electrical Engineering from KTH Royal Institute of Technology, Stockholm, Sweden, and the Ph.D. degree in Control Systems from Chalmers University of Technology, Gothenburg Sweden, in 2004, 2011, and 2016 respectively.

Dr. Altaf has been involved in various R\&D and product development activities related to mechatronics and automatic control systems for more than 15 years, where last 10 years of experience is mainly focused on battery management systems (BMS). From 2004 to 2008, he worked as a Mechatronics R\&D Engineer on radars in Microwave Engineering Research Laboratory at NUST. From 2010 to 2011, he was a Research Engineer with the Automatic Control department at KTH where he worked on event-triggered control over wireless sensor and actuator networks. From 2011 to 2016, he worked at Chalmers University as a Doctoral Researcher on modelling and optimal control of reconfigurable battery systems. Since 2016, he has been working with the main focus on BMS in Automotive industry where he held several positions including Lead Control System Engineer in the department of Advanced Battery Technology at NEVS Sweden (former Saab Cars) and Principal Researcher and Control System Architect at Volvo Group Sweden.

$\mathrm{He}$ is currently working as Chief Engineer at Electromobility department of Volvo Group Sweden where he is leading various R\&D activities related to advanced BMS technologies for range of heavy-duty commercial vehicles. His current $R \& D$ interests are at the intersection of traction batteries, control engineering, power electronics, embedded systems, and system engineering with special focus on model-based controls for automotive applications.

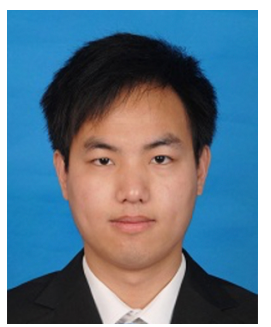

Changfu Zou (Member, IEEE) received the B.E. degree in automotive engineering from the Beijing Institute of Technology, Beijing, China, in 2011 and the $\mathrm{Ph} . \mathrm{D}$. degree in automation and control engineering from the University of Melbourne, Melbourne, VIC, Australia, in 2017.

$\mathrm{He}$ was a visiting student researcher with the Energy, Controls \& Applications Lab, University of California, Berkeley, Berkeley, CA, USA, from 2015 to 2016. Since 2017, he has joined the Automatic Control Group, Chalmers University of Technology, Gothenburg, Sweden, where he was as a Postdoctoral Researcher and currently is an Assistant Professor. His current research interests include modeling and control of energy storage systems for automotive and power grid applications.

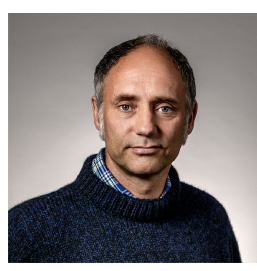

Torsten Wik (Member, IEEE) is a full Professor and the head of Automatic Control at the Department of Electrical Engineering, Chalmers University of Technology, Sweden. He received a M.Sc. in Chemical Engineering, a Licentiate of Engineering degree in Control Engineering in 1996, a Ph.D. in Environmental Sciences in 1999 (majoring in Automatic Control), and a Docent degree in 2004, all degrees from Chalmers University of Technology. From 2005 to 2007 he worked as a senior researcher at Volvo Technology in control system design. For the last decade he has led a growing group of researchers on battery management systems. 